\title{
Local time features of geomagnetic jerks
}

\author{
Hiromichi Nagao ${ }^{1}$, Toshihiko Iyemori ${ }^{2}$, Tomoyuki Higuchi ${ }^{3}$, Shin'ya Nakano ${ }^{1}$, and Tohru Araki ${ }^{1}$ \\ ${ }^{1}$ Department of Geophysics, Graduate School of Science, Kyoto University, Kyoto 606-8502, Japan \\ ${ }^{2}$ Data Analysis Center for Geomagnetism and Space Magnetism, Graduate School of Science, Kyoto University, Kyoto 606-8502, Japan \\ ${ }^{3}$ The Institute of Statistical Mathematics, Tokyo 106-8569, Japan
}

(Received June 7, 2001; Revised October 22, 2001; Accepted November 20, 2001)

\begin{abstract}
The geomagnetic jerk amplitudes, which are defined as abruptness of changes in the trends of geomagnetic time series, are investigated with geomagnetic monthly means computed from hourly mean values at each local time. A statistical time series model in which the trend component is expressed by a second order spline function with variable knots is constructed for each time series. The optimum parameter values of the model including positions of knots are estimated by the maximum likelihood method, and the optimum number of parameters including the number of knots are determined based on the Akaike Information Criterion (AIC). The jerks are detected objectively and automatically by regarding the optimized positions of knots as the occurrence epochs. This analysis reveals that the spatial distributions of jerk amplitudes essentially do not depend on the local time, which indicates that the jerks cannot be explained by abrupt changes in intensities of latitudinally flowing external currents such as the field-aligned currents. Longitudinally flowing currents, on the other hand, such as the ring current could explain the distributions. The abrupt changes of the ring current intensity are estimated from the distributions of jerk amplitudes in the eastward component in 1969, 1978, and 1991 supposing that an abrupt change in the ring current intensity causes a jerk. However those estimated changes cannot consistently explain the distributions of the jerks in the northward and downward components. Therefore it is plausible that the jerks which occurred in 1969,1978 , and 1991 are not caused by external sources but internal ones. It is also confirmed that the occurrence epochs of jerks in the southern hemisphere are a few years after those of the 1969 and 1978 jerks in the northern hemisphere, and it is also found that the jerk in the southern hemisphere occurred a few years after the occurrence of the 1991 jerk in Europe. Taking these time lags in occurrence epochs into account, it can be said that the 1969, 1978 , and 1991 jerks are global phenomena.
\end{abstract}

\section{Introduction}

A geomagnetic secular variation observed on the ground is believed to reflect the motion of the metallic fluid in the outer core and the distribution of the mantle conductivity. It also contains many external contributes such as magnetopause currents, ionospheric currents, ring current, tail current, and field-aligned currents (FACs).

An interesting phenomenon exists in the geomagnetic secular variation whether its source is internal or external is still controversial. Courtillot et al. (1978) pointed out that the trends of the time derivative of geomagnetic secular variations at European region changed suddenly around 1969, which is clearly seen in the $Y$ component as shown in Figs. 1 and 2. Malin and Hodder (1982) termed this phenomenon a geomagnetic jerk, which means mathematically that the secular variation has an impulse in its third order time derivative. Other jerks were reported to have occurred in 1912, 1925, 1978 (e.g., Nevanlinna and Sucksdorff, 1981), 1991 (e.g., Cafarella and Meloni, 1995; Macmillan, 1996), and most recently in 1999 (Mandea et al., 2000). The occurrences of old jerks before 1957 (IGY) are not necessarily definitive because of the small number of observatories

Copy right (C) The Society of Geomagnetism and Earth, Planetary and Space Sciences (SGEPSS); The Seismological Society of Japan; The Volcanological Society of Japan; The Geodetic Society of Japan; The Japanese Society for Planetary Sciences. available and of quite noisy data. Although the jerks had been regarded as sudden changes in the trends of secular variations, Alexandrescu et al. (1995) and Alexandrescu et al. (1996) proposed a general expression for the jerk signals and suggested that a jerk is not just the change in the secular variation trends but also the behavior of the field over the next decade.

There are many discussions about the origin of the jerks. Malin and Hodder (1982) evaluated the spherical harmonic coefficients from the third order time derivative of the geomagnetic annual means at 83 observatories, and concluded that the 1969 jerk is of internal origin. Alldredge (1984) and Alldredge (1985), however, questioned the data selection and the method of analysis by Malin and Hodder (1982), and insisted that an external current system could generate the observed jerks. Despite of many researches on the jerks, whether their sources are internal or external is still controversial (e.g., Kerridge and Barraclough, 1985; McLeod, 1985; Gavoret et al., 1986; Gubbins and Tomlinson, 1986; Thompson and Cain, 1987; Whaler, 1987; Golovkov et al., 1989; McLeod, 1992). It is suggested that the jerks may have some correlations with abrupt changes in decadal length-of-day variation (e.g., Courtillot et al., 1978; Le Mouël and Courtillot, 1981; Davis and Whaler, 1997; Mandea et al., 2000) and with motion of fluid flow at the top 

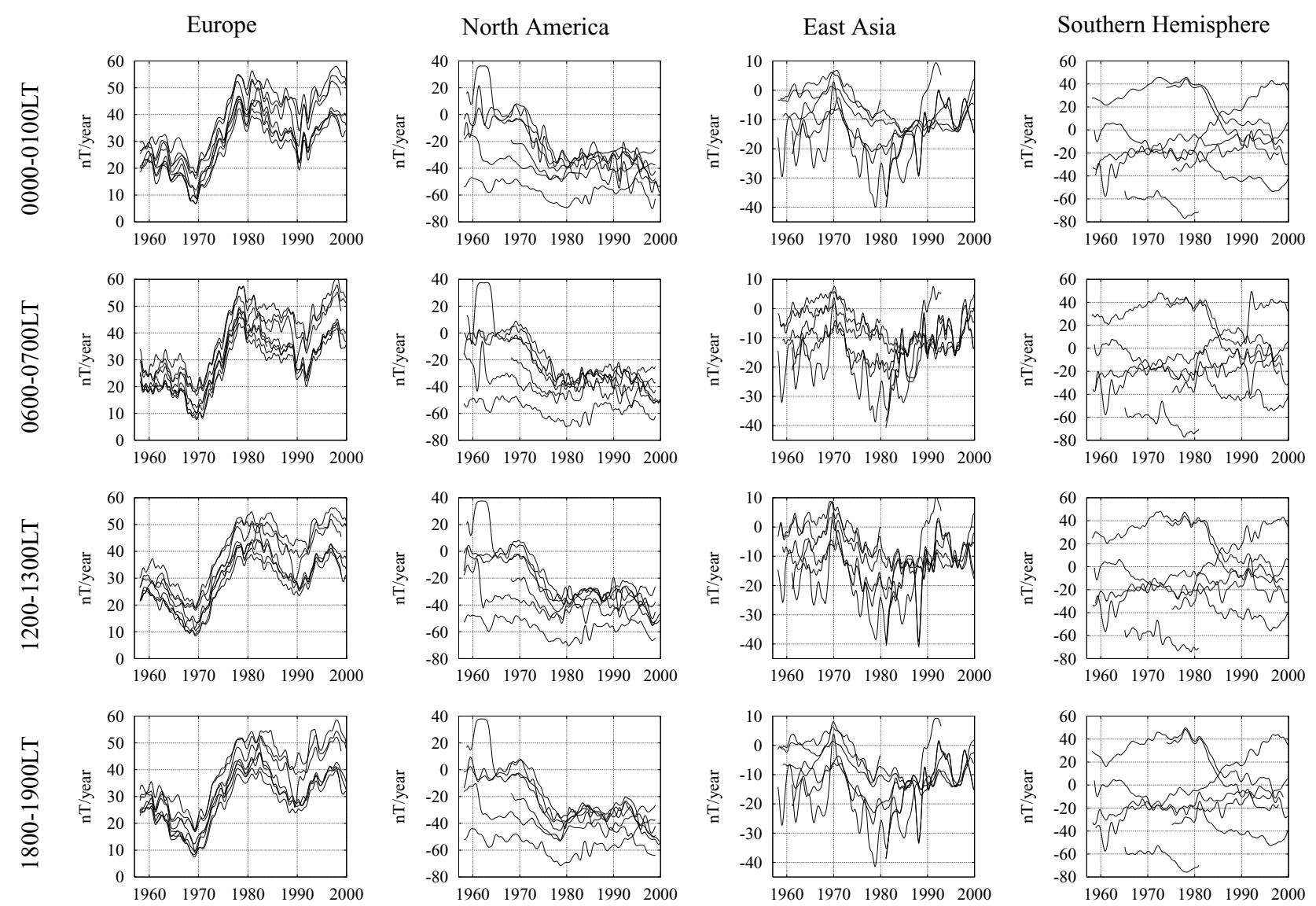

Fig. 1. The time derivative of geomagnetic monthly means of the $Y$ component at 0000-0100 LT, 0600-0700 LT, 1200-1300 LT, and 1800-1900 LT. The trends especially in European observatories sometimes change suddenly in several years around 1969, 1978, and 1991. These phenomena are called geomagnetic jerks.

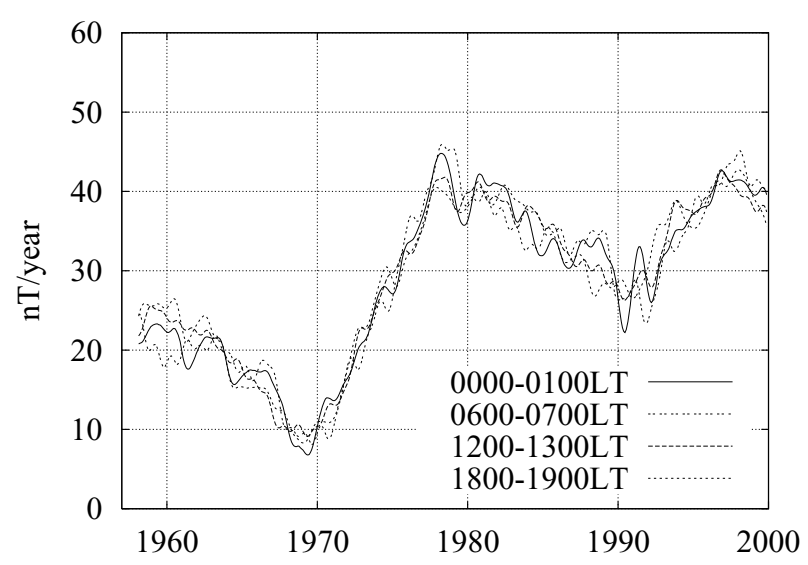

Fig. 2. The time derivative of the secular variations at Wingst, Germany obtained from monthly means for 0000-0100 LT, 0600-0700 LT, 1200-1300 LT, and 1800-1900 LT.

of the core (e.g., Le Huy et al., 1998, 2000). One of the motivations to clarify the source of the jerks is that they could give a constraint to the distribution of the mantle conductivity if they are of the core origin, the upper bound value of the magnitude of the lower mantle conductivity was estimated by many papers (e.g., Achache et al. , 1980; Ducruix et al.,
1980; Backus, 1983; Mandea Alexandrescu et al., 1999).

There are also some discussions in spatial and temporal distributions of the jerks. The 1969 and 1978 jerks are confirmed to be global phenomena by many papers (e.g., Le Mouël et al., 1982; Alexandrescu et al., 1996), and the 1991 jerk has worldwide character (e.g., De Michelis et al., 1998; Le Huy et al., 1998, 2000). It was also reported that the occurrences of the jerks in the southern hemisphere are a few years after those of the 1969 and 1978 jerks in the northern hemisphere (e.g., Alexandrescu et al., 1996), and the occurrence of the jerks in North and South America is a few years before that of the 1991 jerk in other regions (e.g., De Michelis et al., 1998). These commonly observed time lags in occurrence epochs could be evidences, which indicate that the jerks are of internal origin.

The previous studies on the jerks used geomagnetic annual means or monthly means averaged over all hours and all days, which lose the information of local time dependences. In this paper, the jerks are investigated with monthly means computed from hourly mean values at each local time. The magnetic field lines penetrating to the high conductive outer core move with the metallic fluid motion as explained by the frozen-in flux theory. Because it can be considered that the outer core corotates with the mantle in decadal time scale, phenomena of the core origin observed on the ground should 
show the regionality rather than the local time dependence. The magnetic fields of external origin, on the other hand, contribute to the geomagnetic field with the local time dependence because the external current system is fixed in the solar-terrestrial system and is essentially independent of the rotation of the earth. Therefore it is important to investigate the local time dependences of jerks to determine whether the sources of the jerks are internal or external.

The data description shall be mentioned in Section 2 and the method of our analysis is developed in Section 3. A statistical time series model is applied to the monthly means at each local time and they are decomposed into trend, seasonal, stationary autoregressive (AR), and observational noise components. The model parameters are optimized by the maximum likelihood method and the best model is selected based on the Akaike Information Criterion (AIC). The occurrence epochs of jerks are determined automatically based on the statistical time series model. The results of the analysis are shown in Section 4. Occurrence rates of jerks and spatial and temporal distributions of jerk amplitudes in the $X, Y$, and $Z$ components are shown at each local time. Then whether the origin of the jerks is internal or external is discussed in Section 5.

\section{Data}

We use geomagnetic hourly mean values obtained at observatories distributed worldwide. The data are collected through the World Data Center system (e.g., see http: / / swdcdb.kugi.kyoto-u.ac.jp/). The time series at an observatory should be continuous for more than ten years, since a geomagnetic jerk is a sudden change in the trends of geomagnetic secular variation. A missing period shorter than about one year is accepted, and it is unnecessary to be interpolated before the analysis because all components except for an observational noise component in a statistical model can be estimated by the Kalman filter algorithm even for the missing period (Kitagawa and Gersch, 1996) as mentioned in the next section. The time series of 124 geomagnetic observatories are selected according to the criteria mentioned above. The distribution of these observatories is shown in Fig. 3. We use the time series from 1957 (IGY) to 1999 but do not before the IGY, for the worldwide coverage of the observatories available is not enough. The period of data available at each observatory is shown in Fig. 4. Obvious artificial spikes and baseline jumps in the data are corrected manually before the analysis. Unfortunately small errors which would affect the result of statistical time series analysis may remain even after this manual correction. However, we can avoid artifacts due to such small errors by a careful check for the results based on the comparison of the results with those obtained at other observations as shown in Section 4.

A vector of geomagnetic hourly mean value at the $i$-th observatory $O_{i}$ is expressed as $\boldsymbol{B}_{n, d, h}\left(O_{i}\right)=\left(X_{n, d, h}\left(O_{i}\right)\right.$, $\left.Y_{n, d, h}\left(O_{i}\right), Z_{n, d, h}\left(O_{i}\right)\right)^{T}$, where $n$ is the consecutive month from January 1957, $d$ is the day of the month, and $h(h=$ $1,2, \ldots, 24)$ is the local time. The superscript $T$ denotes the transpose operation. Hourly means at $h=1,2, \ldots$, and 24 correspond to the averages in the periods 0000-0100 LT, 0100-0200 LT, ..., and 2300-2400 LT, respectively. The

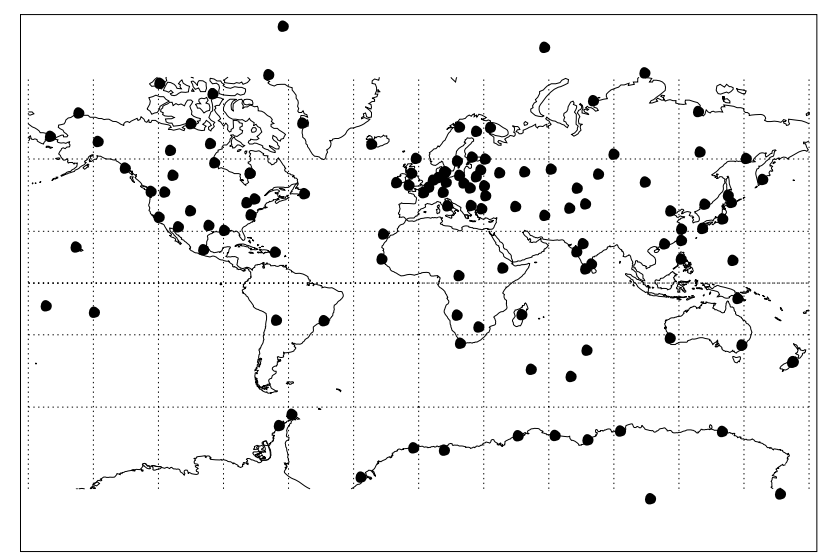

Fig. 3. The distribution of the 124 geomagnetic observatories whose data are used in this paper.

monthly means of each local time are computed from the hourly means by

$$
\overline{\boldsymbol{B}}_{n, h}\left(O_{i}\right)=\frac{1}{D_{n}} \sum_{d=1}^{D_{n}} \boldsymbol{B}_{n, d, h}\left(O_{i}\right),
$$

where $D_{n}$ is the number of days of the month $n$.

\section{Method of Analysis}

\subsection{Statistical time series model}

Most of the papers assume that the geomagnetic jerks occurred around 1969, 1978, and 1991, and then obtain the jerk amplitudes. Their methods, however, are subjective in the determination of the occurrence epochs of jerks, and the estimated jerk amplitudes may not be reliable as has been pointed out by, for example, Alldredge (1984). Stewart and Whaler (1995) and Alexandrescu et al. (1995) developed the objective methods with the optimal piecewise regression analysis and the wavelet analysis, respectively, for determining the occurrence epochs of jerks. The former method was applied to geomagnetic annual means, but it is better to adopt monthly means rather than annual means for jerk analyses, because the abrupt change occur only in a few years as seen in Figs. 1 and 2, and the temporal resolution of annual means may not be sufficient to analyze the jerk. The latter method, on the other hand, was applied to geomagnetic monthly means and succeeded in the objective determination of jerk epochs. Alexandrescu et al. (1995) and Alexandrescu et al. (1996) proposed a general expression for a jerk signal

$$
j(t)=\beta H\left(t-t_{0}\right)\left(t-t_{0}\right)^{\alpha},
$$

where $t_{0}$ is the jerk epoch, $\alpha$ is the regularity, $\beta$ is the amplitude, and $H(t)$ is the Heaviside function. Alexandrescu et al. (1995) and Alexandrescu et al. (1996) applied the wavelet analysis to geomagnetic data, and evaluated the regularity to be closer 1.5 rather than to 2 . This may indicate that a model with non-integer $\alpha$ value can be applicable to the trend component, but the second order spline function is adopted in this paper otherwise physical interpretation of 

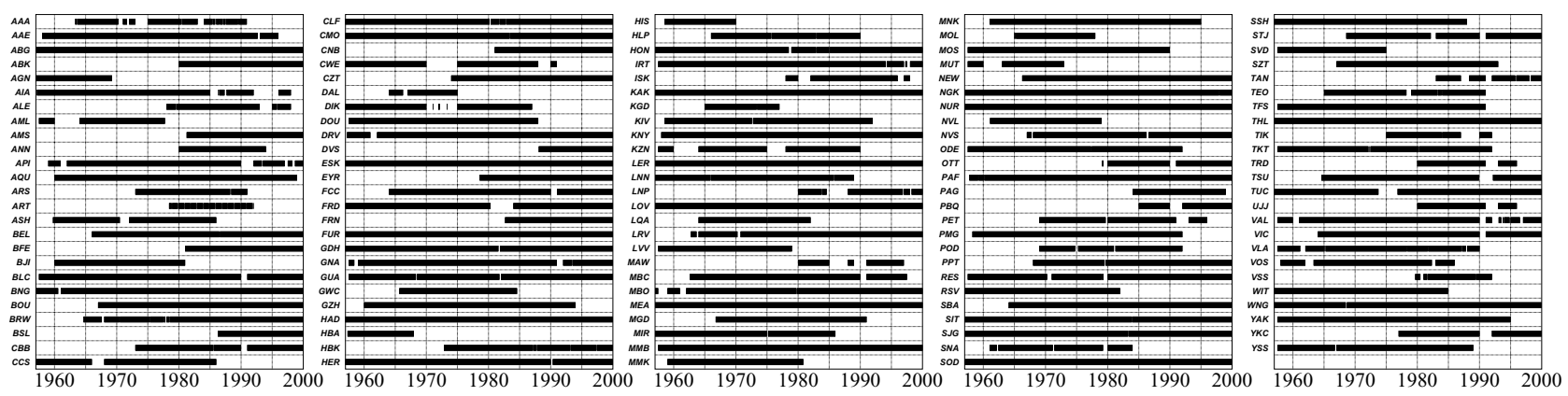

Fig. 4. The periods of geomagnetic hourly values used in this paper are indicated with horizontal bars. The abbreviation codes of the geomagnetic observatories are shown on the left.

results of the analysis mentioned in Section 5 would be difficult.

In this paper, a statistical time series model, in which the seasonal adjustment and short time scale adjustment are taken into account, is applied to geomagnetic monthly means to derive the trend component of the geomagnetic field. A geomagnetic time series of monthly means in a component $E_{n}$ (i.e., $E_{n}=X_{n}, Y_{n}$, or $Z_{n}$; for simplicity, the bar, the subscript $h$, and $O_{i}$ in the Eq. (1) are omitted hereafter except for the cases necessary to be emphasized) is described by the following statistical time series model:

$$
E_{n}=t_{n}+s_{n}+p_{n}+w_{n},
$$

where $t_{n}$ is the trend component, $s_{n}$ is the seasonal component, $p_{n}$ is the stationary $\mathrm{AR}$ component, and $w_{n}$ is the observational noise.

3.1.1 Trend component The time derivative of decadal geomagnetic variation is approximated by a curve which consists of several jagged peaks as seen in Figs. 1 and 2 . The trend component $t_{n}$ of the geomagnetic time series is expressed by the second order spline function because a curve of the time derivative of geomagnetic time series can be approximated by a first order spline function with several knots $\xi_{1}, \xi_{2}, \ldots, \xi_{K}$, where $K$ is the number of knots. The position of a knot corresponds to the occurrence epoch of a jerk. Here each interval between successive knots is assumed to be more than five years to avoid too many knots concentrating in a short period, and each interval between an endpoint of the time series and the adjacent knot is kept more than three years. Stewart and Whaler (1995) adopted the similar condition.

The trend component expressed by the second order spline function can be represented by the following model:

$$
t_{n}=t_{n-1}+\delta t_{n-1}+\frac{1}{2} \delta^{2} t_{n-1} .
$$

The first order difference component $\delta t_{n}$ and the second order difference component $\delta^{2} t_{n}$ in the Eq. (4) satisfy

$$
\begin{aligned}
\delta t_{n} & =\delta t_{n-1}+\delta^{2} t_{n-1} \\
\delta^{2} t_{n} & =\delta^{2} t_{n-1}+v_{n 1},
\end{aligned}
$$

where $v_{n 1}$ is the system noise which obeys the normal distribution function. The mean of $v_{n 1}$ is zero and the variance is $\tau_{1}^{2}$, i.e., $v_{n 1} \sim N\left(0, \tau_{1}^{2}\right) . \tau_{1}^{2}$ has a large value at every knot where the second order time derivative of the spline function is not continuous while the value of $\tau_{1}^{2}$ is zero at other time. The values of $\tau_{1}^{2}$ at the knots $\xi_{1}, \xi_{2}, \ldots, \xi_{K}$ are written as $\tau_{11}^{2}, \tau_{12}^{2}, \ldots, \tau_{1 K}^{2}$, respectively. The parameters which should be optimized in the trend component are the number and the positions of the knots and the variances of the system noise at each knot.

3.1.2 Seasonal component The seasonal component $s_{n}$, which represents the annual variation in the data, should have a twelve months periodicity, i.e., $s_{n} \approx s_{n-12}$. This condition can be rewritten as

$$
\sum_{i=0}^{11} s_{n-i}=v_{n 2},
$$

where $v_{n 2}$ is the system noise which obeys $N\left(0, \tau_{2}^{2}\right)$ (Kitagawa and Gersch, 1996). The value of $\tau_{2}^{2}$ is optimized later.

3.1.3 Stationary autoregressive component It is not easy to explain the physical meaning of the stationary AR component $p_{n}$. If this component is not included in the model (3), the best model tends to have more knots than it is expected as shown in the next section. This component may represent short time scale variations less than one year, i.e., the solar effects, or the induction responses to the external field variation which reflect the mantle conductivity structures. The stationary AR component can be expressed as

$$
p_{n}=\sum_{i=1}^{m} a_{i} p_{n-i}+v_{n 3},
$$

where $m$ is the AR order of this component and $v_{n 3}$ is the system noise which obeys $N\left(0, \tau_{3}^{2}\right)$. The value of $\tau_{3}^{2}$ is optimized later.

3.1.4 Observational noise The observational noise $w_{n}$ is assumed to be a white noise. It obeys the normal distribution function whose mean is zero and variance is $\sigma^{2}$, i.e., $w_{n} \sim N\left(0, \sigma^{2}\right)$. The value of $\sigma^{2}$ is also optimized later.

\subsection{Model optimization}

The parameter vector involved in the Eqs. (3)-(8)

$$
\begin{aligned}
\boldsymbol{\theta}= & \left(\xi_{1}, \xi_{2}, \ldots, \xi_{K}, a_{1}, a_{2}, \ldots, a_{m},\right. \\
& \left.\tau_{11}^{2}, \tau_{12}^{2}, \ldots, \tau_{1 K}^{2}, \tau_{2}^{2}, \tau_{3}^{2}, \sigma^{2}\right)
\end{aligned}
$$


is estimated simultaneously by the maximum likelihood method. The optimum parameter vector $\hat{\boldsymbol{\theta}}$ (The hat means "optimum") that makes the log-likelihood function $\ell(\boldsymbol{\theta})$ maximum should be estimated by a numerical method because it is hard to obtain it analytically. The value of the $\log$-likelihood $\ell(\boldsymbol{\theta})$ for a given parameter vector $\boldsymbol{\theta}$ can be calculated simply by the Kalman filter algorithm through the state space model (Anderson and Moore, 1979). The state space model can be constructed as

$$
\begin{aligned}
& \boldsymbol{x}_{n}=F \boldsymbol{x}_{n-1}+G \boldsymbol{v}_{n} \\
& E_{n}=H \boldsymbol{x}_{n}+w_{n}
\end{aligned}
$$

from the Eqs. (3)-(8) by defining the state space vector $\boldsymbol{x}_{n}$ and the system noise vector $\boldsymbol{v}_{n}$ as

$$
\begin{aligned}
& \boldsymbol{x}_{n}=\left(\begin{array}{llllll}
t_{n} & \delta t_{n} & \delta^{2} t_{n} s_{n} & s_{n-1} & \cdots & s_{n-10}
\end{array}\right. \\
& \left.\begin{array}{llll}
p_{n} & p_{n-1} & \cdots & p_{n-m+1}
\end{array}\right)^{T} \\
& \boldsymbol{v}_{n}=\left(\begin{array}{lll}
v_{n 1} & v_{n 2} & v_{n 3}
\end{array}\right)^{T},
\end{aligned}
$$

and the matrices $F, G$, and $H$ as

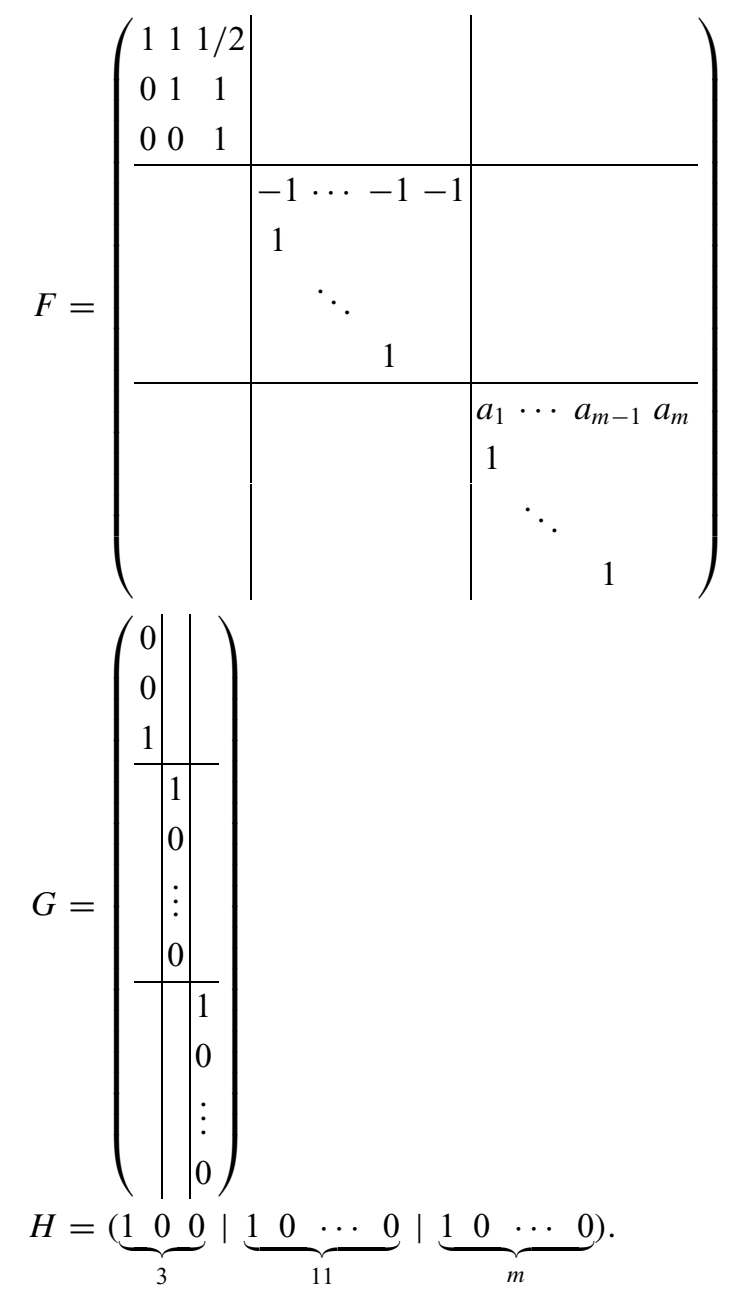

First an appropriate initial parameter vector $\boldsymbol{\theta}_{0}$ is given and the log-likelihood function $\ell\left(\boldsymbol{\theta}_{0}\right)$ is calculated by the Kalman filter. Then the parameter vector is updated by the quasi-Newton method (Press et al., 1986) to make the log-likelihood larger. This procedure is iterated until the parameter vector $\hat{\boldsymbol{\theta}}$ makes the log-likelihood maximum. The AIC

$$
\begin{aligned}
\mathrm{AIC} & =-2 \ell(\hat{\boldsymbol{\theta}})+2 \operatorname{dim} \hat{\boldsymbol{\theta}} \\
& = \begin{cases}-2 \ell(\hat{\boldsymbol{\theta}})+2(2 K+2) & (m=0) \\
-2 \ell(\hat{\boldsymbol{\theta}})+2(2 K+m+3) & (m \neq 0)\end{cases}
\end{aligned}
$$

is employed to select the best model among the models with different number of the parameters. The model which makes the AIC minimum is assumed to be the best model for the time series. See Higuchi and Ohtani (2000) for an application of the AIC to determination of the number and positions of the knots. The final estimate of the state vector variable is obtained by the fixed interval smoother algorithm (Kitagawa and Gersch, 1996). The optimum positions of the knots are treated as candidates for jerk occurrence. It should be noted that, because our procedure detects any jerk regardless of its origin (internal or external one), the knot positions determined must undergo further analyses, i.e., an examination of spatial and local time dependency of occurrence rate and jerk amplitude as explained in Sections 4 and 5. As a result, the jerk of external origin and one due to small artificial errors can be excluded. The jerk amplitude $\delta^{3} E_{n}$ at a knot $\xi_{i}$ is defined as:

$$
\delta^{3} E_{\xi_{i}}=\delta^{2} t_{\xi_{i}}-\delta^{2} t_{\xi_{i}-1}
$$

The unit of this jerk amplitude is not nT/year ${ }^{3}$ but nT/year ${ }^{2}$ because it is defined as a simple difference between successive second order difference components. The similar definition of the jerk amplitude (Eq. (18)) is given by the previous papers (e.g., De Michelis et al., 1998).

\section{Results}

An example of the decomposition of geomagnetic time series by the method mentioned in the previous section is shown in Fig. 5. The data used in Fig. 5 are the monthly means of the $Y$ component at Wingst $\left(53.75^{\circ} \mathrm{N}, 9.07^{\circ} \mathrm{E}\right)$, Germany, obtained from 0000-0100 LT. Although there exist missing period for July and August 1968, they can be interpolated automatically by the Kalman filter (Anderson and Moore, 1979). The number of the knots for the best model is $\hat{K}=4$ and they locate at $\hat{\xi}_{1}=$ December $1964, \hat{\xi}_{2}=$ December 1969, $\hat{\xi}_{3}=$ March 1978, and $\hat{\xi}_{4}=$ December 1990, when the jerks are believed to have occurred except for 1964. Other parameter values for the best model are $\hat{m}=2, \hat{a}_{1}=1.32 \mathrm{nT}, \hat{a}_{2}=-0.487 \mathrm{nT}, \hat{\tau}_{11}^{2}=1.59 \times 10^{-5}$ $\mathrm{nT}^{2}, \hat{\tau}_{12}^{2}=9.48 \times 10^{-5} \mathrm{nT}^{2}, \hat{\tau}_{13}^{2}=8.18 \times 10^{-5} \mathrm{nT}^{2}, \hat{\tau}_{14}^{2}=$ $1.30 \times 10^{-3} \mathrm{nT}^{2}, \hat{\tau}_{2}^{2}=6.84 \times 10^{-3} \mathrm{nT}^{2}, \hat{\tau}_{3}^{2}=1.00 \times 10^{-2}$ $\mathrm{nT}^{2}$, and $\hat{\sigma}^{2}=10.3 \mathrm{nT}^{2}$. The log-likelihood is -1526.29 and the AIC is 3078.52 . The seasonal component has the amplitude of $\sim 4 \mathrm{nT}$. The number of the knots becomes 5 and the AIC increases to 3126.22 if the stationary AR component $p_{n}$ is not included in the model (3), which indicates an advantage of including the stationary AR component. The same tendency is seen in most of the cases. The amplitudes of $s_{n}, p_{n}$, and $w_{n}$ tend to be amplified and the AR order $m$ tends to increase when the data at high or low latitude observatories are used or when the daytime data are used.

As mentioned in Subsection 3.2, not all of the detected jerks are of internal origin. For example, the optimal knot $\hat{\xi}_{1}$ detected at Wingst may reflect a local phenomenon or a 


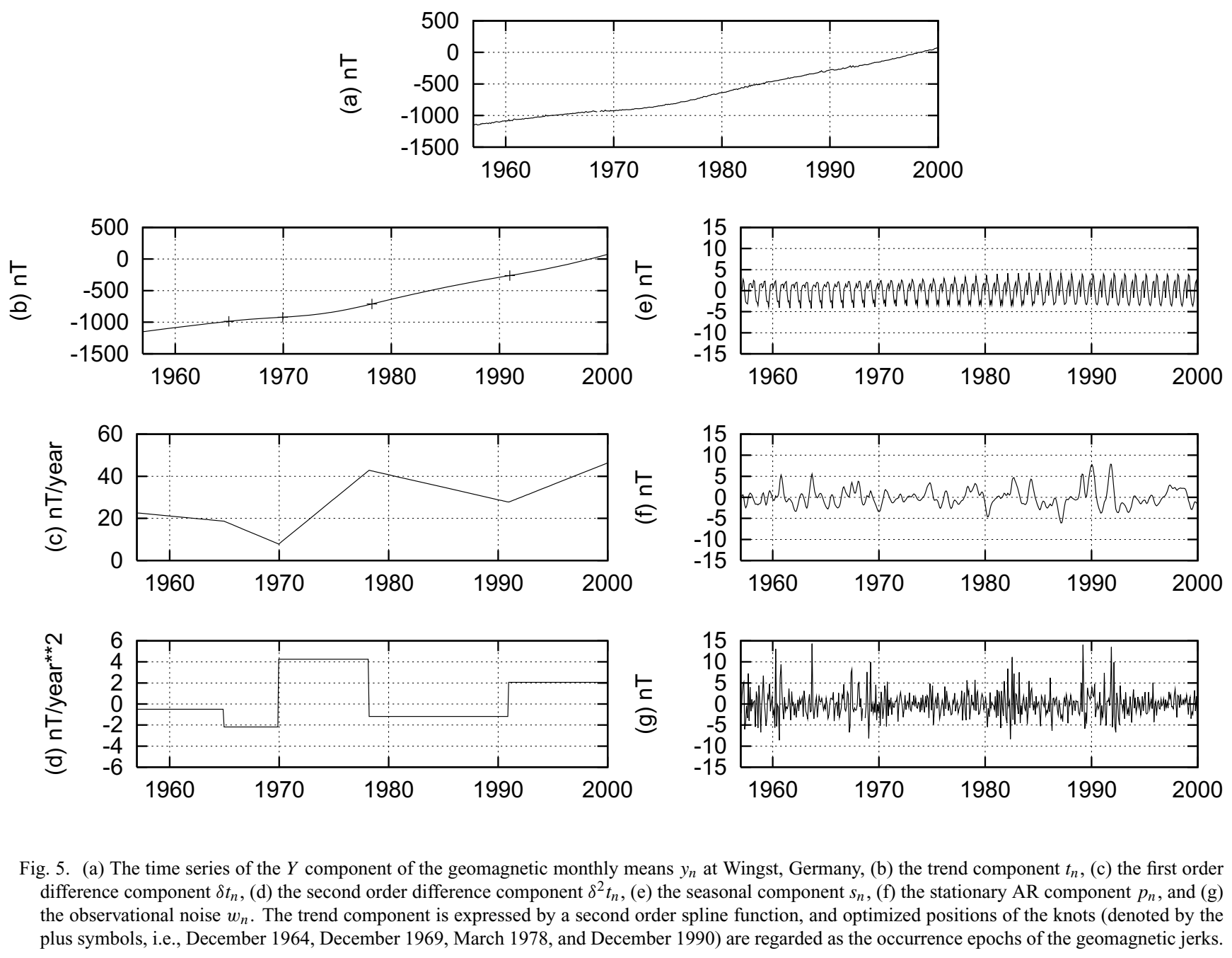

small error in the data. Therefore we examine the dependency of its occurrence rate to evaluate the significance of the knots by looking at the local time dependency. For this purpose, the occurrence rate of jerks is calculated in this section.

The occurrence rate of jerks for a component $E$ in the year $y$ at the local time $h$ is defined by

$$
R_{E, y, h}=\frac{1}{n_{y}} \sum_{i} \sum_{\xi_{j} \in y}\left|\delta^{3} E_{\xi_{j}, h}\left(O_{i}\right)\right|,
$$

where $n_{y}$ is the number of observatories available in the year $y$. Figure 6 shows the occurrence rates of jerks for each component detected by our method. It can be confirmed that the $R_{Y, y, h}$ (i.e., $R_{E, y, h}$ for $Y$ component) has clear peaks for each local time in 1969 and 1977, when the jerks are believed to have occurred (e.g., Courtillot et al., 1978), and around 1990 for all, 1200-1300 LT, and 1800-1900 LT periods. The $R_{Y, y, h}$ has also small peaks in 1982 and 1993 when jerks are not reported to have occurred. The $R_{X, y, h}$ (i.e., $R_{E, y, h}$ for $X$ component) has clear peaks in 1961, 1966, and 1995, and small peaks in 1970, 1977, 1983, and 1988. The $R_{Z, y, h}$ (i.e., $R_{E, y, h}$ for $Z$ component) has clear peaks in 1963, 1970, 1978, 1983, 1988, and 1993. The occurrence rate for each component (i.e., $R_{X, y, h}, R_{Y, y, h}$, and $R_{Z, y, h}$ ) has peaks almost in the same years with time lags, if any, of one or two years. These years, when the occurrence rates have peaks (i.e., 1961, 1966, 1969, 1977, 1983, 1988, and 1993), are the candidates of occurrence epoch of jerks of internal origin. To identify an origin of the detected jerks, namely, internal or external one, further analysis in terms of geophysical viewpoint is required. Thus, we investigate the global distributions of the jerk amplitudes $\delta^{3} X_{n, h}, \delta^{3} Y_{n, h}$, and $\delta^{3} Z_{n, h}$. Figures 7, 8 , and 9 demonstrate the spatial distributions of jerk amplitudes for each component in 00000100 LT, 0600-0700 LT, 1200-1300 LT, and 1800-1900 LT around the years when the occurrence rate has a peak. It can be seen in these figures that the jerk amplitudes do not essentially depend on the local time.

\section{Discussion}

\subsection{Can external currents explain the observed jerks?}

In this section, we discuss whether the local time independent feature of the jerk amplitudes mentioned in the previous section can be generated by an abrupt change of external currents or not. We assume that the influence of induced currents to a jerk amplitude is negligible and that the structure of external current system is fixed in the solar-terrestrial system. Meanwhile, as mentioned in Section 1, Alexandrescu et al. (1995) and Alexandrescu et al. (1996) proposed that a 
$\mathrm{X}$
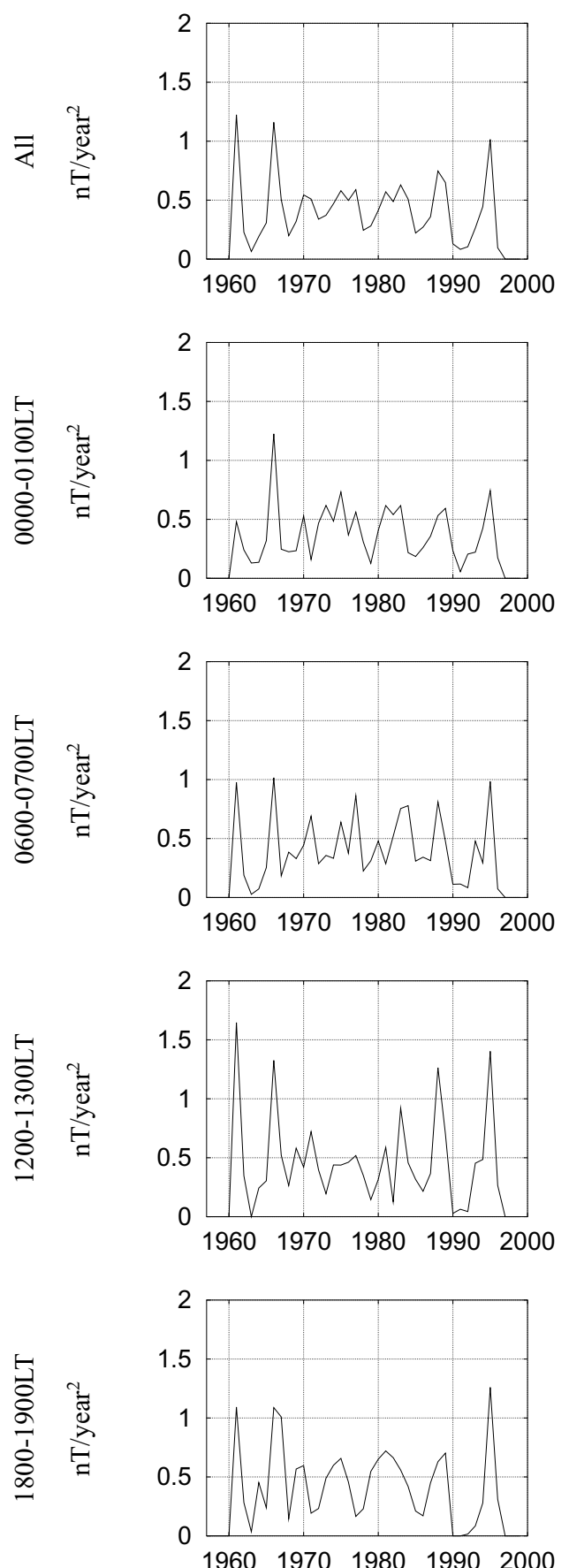

$\mathrm{Y}$
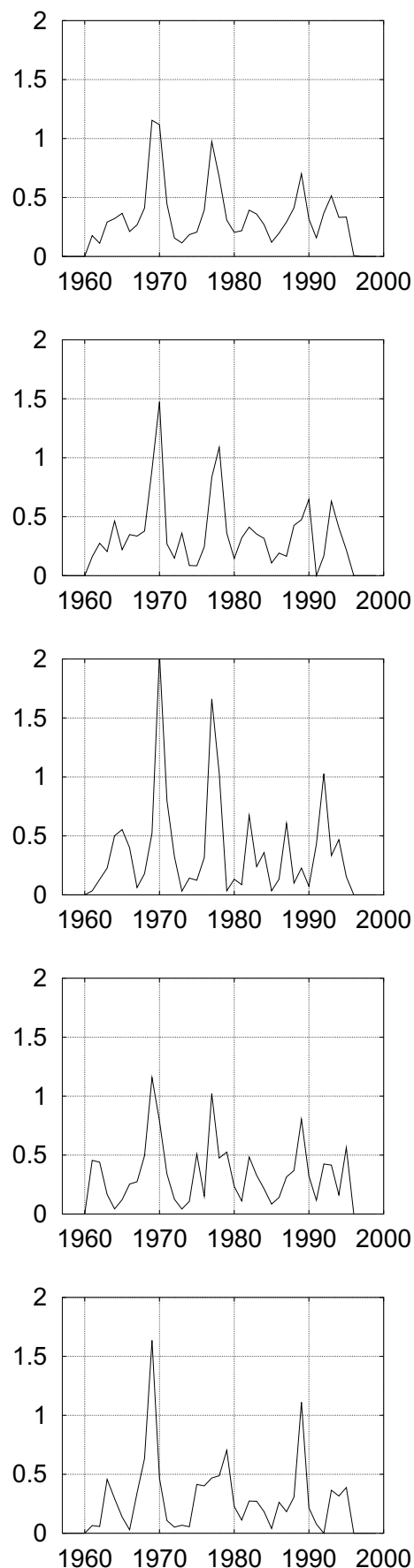

Z
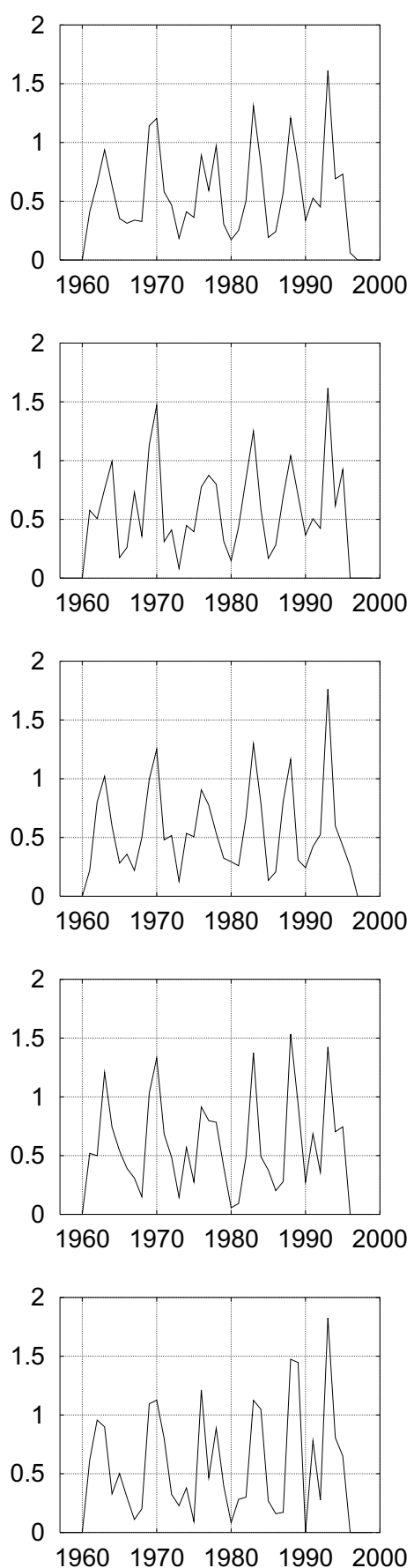

Fig. 6. The occurrence rate of geomagnetic jerks for each component obtained from all of the local time (the top three panels) and at each local time. The occurrence rate is defined by Eq. (19).

jerk is not just the change in the secular variation trend but also the behavior of the field over the next decade. However we discuss the results based on classical definition of jerks, i.e., "a jerk is a sudden change in secular variation trends."

5.1.1 Possibility of field-aligned current as the origin of jerk As a simple example, Fig. 10(a) shows a pair of FACs which produce magnetic disturbance on the ground. An FAC (denoted as FAC1) flows into the dawn side of the ionosphere at high latitude region, and another one (denoted as FAC2) flows out from the dusk side of the ionosphere.
The current intensities of FAC1 and FAC2 must be equal $(=J)$ in order to keep the current continuity. FAC1 generates westward geomagnetic field $Y_{1}$ at a mid-latitude observatory while FAC2 generates eastward field $Y_{2}$. Assuming that $J$ varies as $J=J_{0} e^{i \omega t}$, i.e., $Y_{1}$ and $Y_{2}$ vary as $Y_{1}=-Y_{0} e^{i \omega t}$ and $Y_{2}=Y_{0} e^{i \omega t}$, respectively, we can easily obtain $\delta^{3} Y_{1}=-\delta^{3} Y_{2}$; the signs of the jerk amplitudes are opposite. Generally speaking, a jerk amplitude should depend on the local time not only in its magnitude but also in its sign if FACs are the origins of the jerks. 

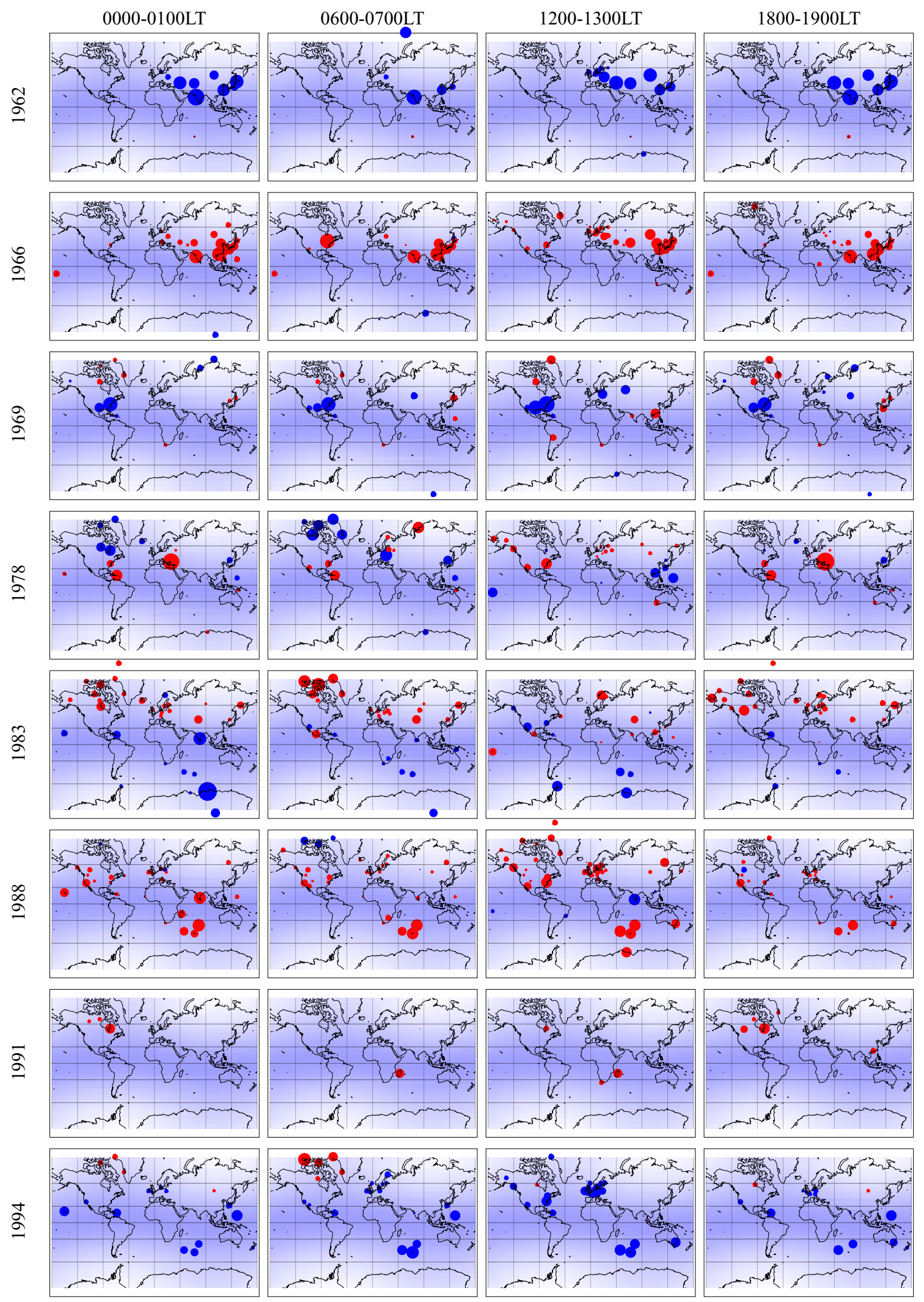

Fig. 7. The distributions of the jerk amplitudes for the $X$ component $\delta^{3} X$ in periods 1961-1963, 1965-1967, 1968-1970, 1977-1979, 1982-1984, 1987-1989, 1990-1992, and 1993-1995 obtained from the data at 0000-0100 LT, 0600-0700 LT, 1200-1300 LT, and 1800-1900 LT. The blue circles show positive values and the red ones show negative values. The magnitude of the jerk amplitudes is represented by the radius of the circles. The background color contour indicates the effects of the westward ring current in the $X$ component estimated by a simple model calculation. 

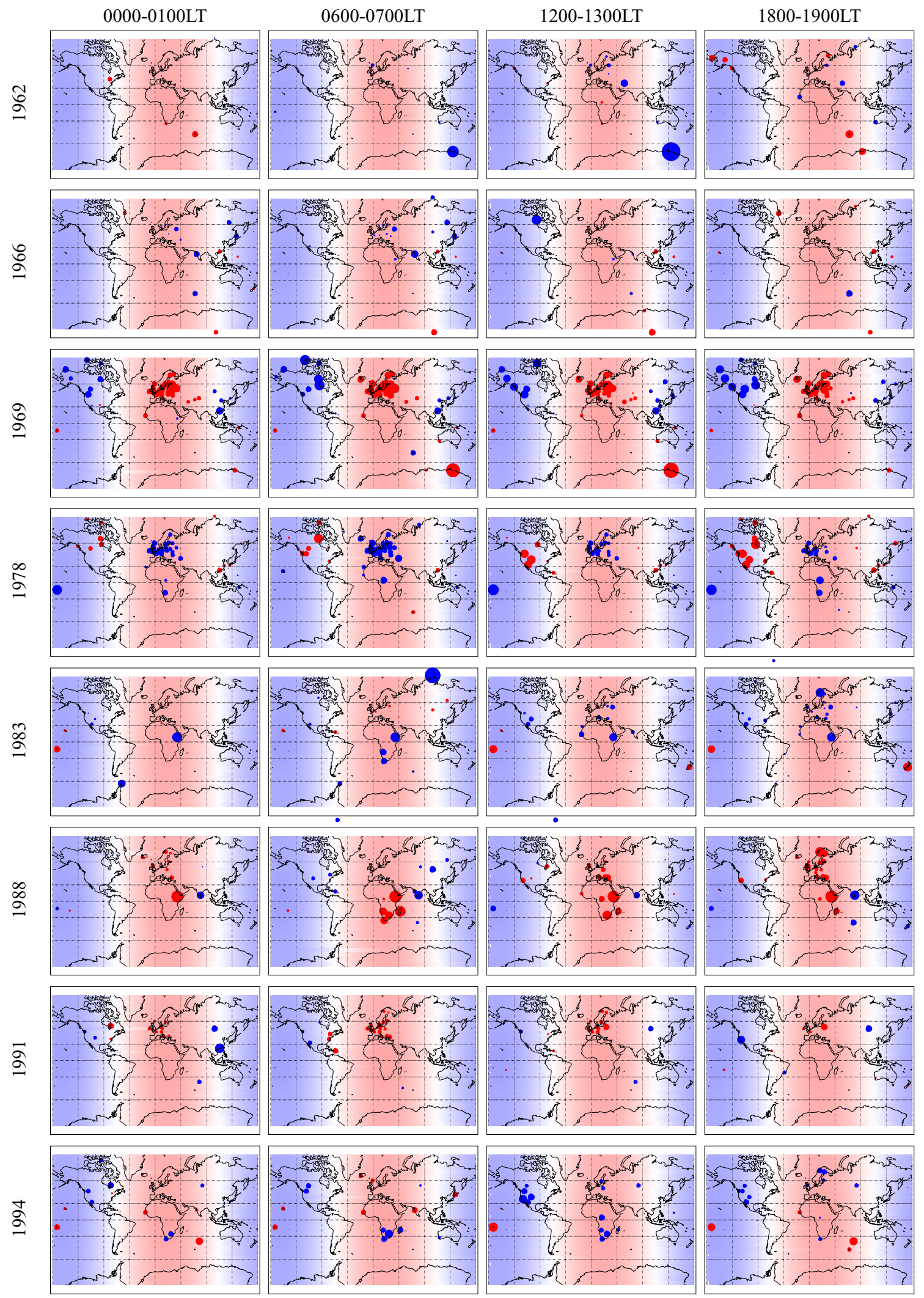

Fig. 8. The distributions of the jerk amplitudes for the $Y$ component $\delta^{3} Y$ in periods 1961-1963, 1965-1967, 1968-1970, 1977-1979, 1982-1984, 1987-1989, 1990-1992, and 1993-1995 obtained from the data at 0000-0100 LT, 0600-0700 LT, 1200-1300 LT, and 1800-1900 LT. The blue circles show positive values and the red ones show negative values. The magnitude of the jerk amplitudes is represented by the radius of the circles. The background color contour indicates the effects of the westward ring current in the $Y$ component estimated by a simple model calculation. 

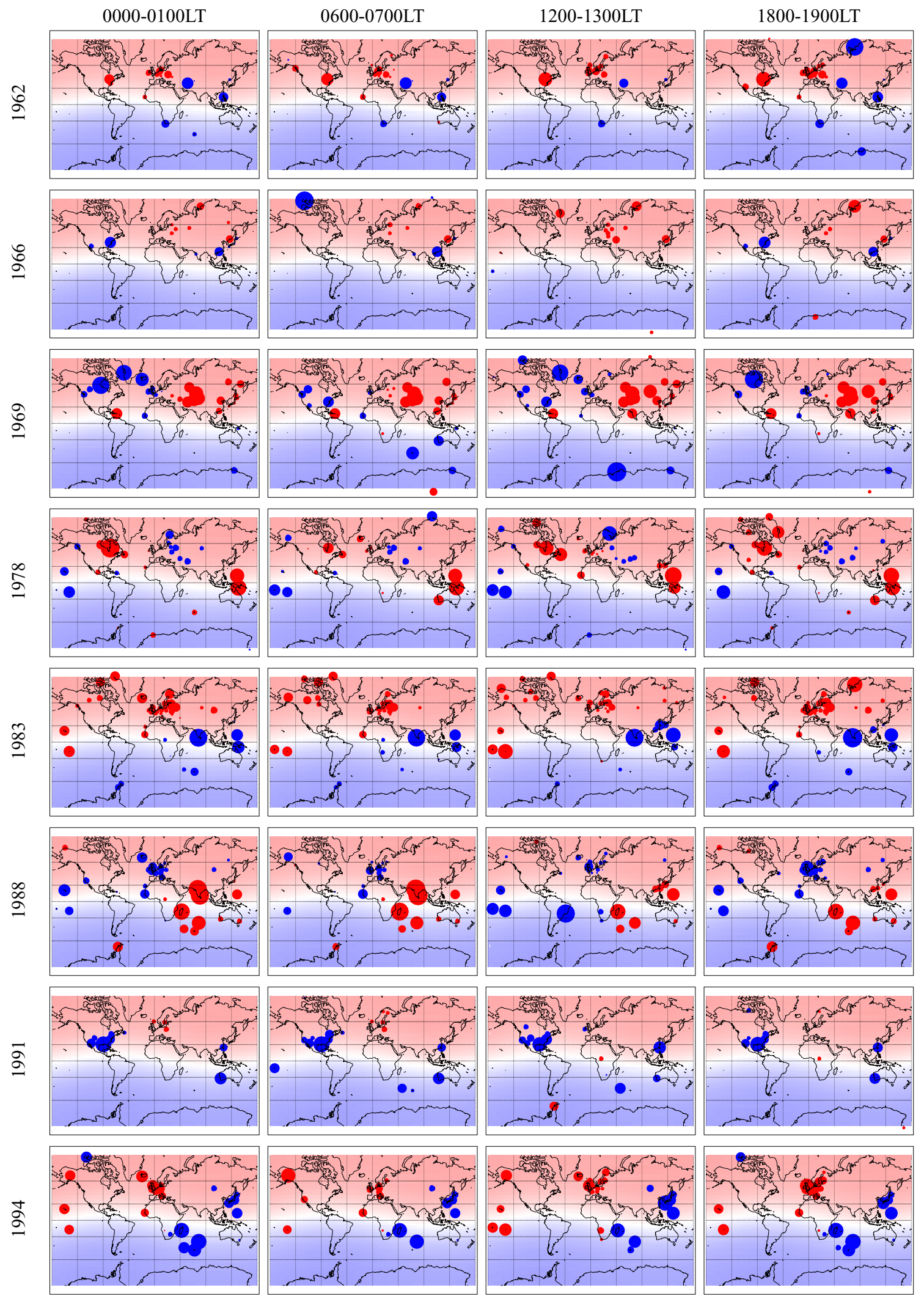

Fig. 9. The distributions of the jerk amplitudes for the $Z$ component $\delta^{3} Z$ in periods 1961-1963, 1965-1967, 1968-1970, 1977-1979, 1982-1984, 1987-1989, 1990-1992, and 1993-1995 obtained from the data at 0000-0100 LT, 0600-0700 LT, 1200-1300 LT, and 1800-1900 LT. The blue circles show positive values and the red ones show negative values. The magnitude of the jerk amplitudes is represented by the radius of the circles. The background color contour indicates the effects of the westward ring current in the $Z$ component estimated by a simple model calculation. 
Table 1. The abrupt changes in the ring current intensity $\delta^{3} J$ estimated from the distributions of the jerk amplitudes of each component shown in Figs. 7 , 8 , and 9 supposing that the jerks are caused by the ring current. The positive values correspond to the eastward changes and negative ones correspond to the westward changes. The unit is ampere/year ${ }^{2}$.

\begin{tabular}{rrrrrrrrr}
\hline & 1962 & 1966 & 1969 & 1978 & 1983 & 1988 & 1991 & 1994 \\
\hline$X$ & -7.76 & 4.81 & -2.45 & -1.86 & -1.67 & 2.52 & 1.29 & -4.41 \\
$Y$ & 10.15 & -0.52 & -10.89 & 10.73 & -6.71 & -4.57 & -15.00 & -0.42 \\
$Z$ & -2.52 & -0.21 & -1.73 & 0.18 & -2.66 & 1.51 & 3.16 & -3.51 \\
\hline
\end{tabular}

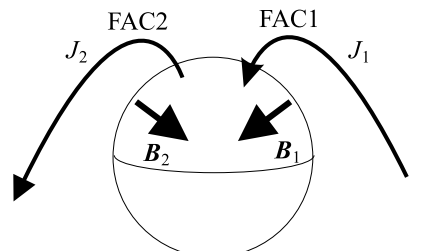

(a)

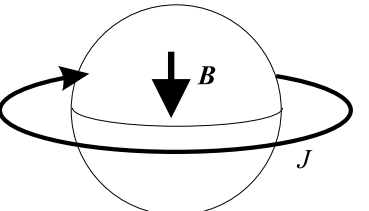

(b)
Fig. 10. (a) A pair of field-aligned currents and (b) a magnetospheric equatorial ring current. Geomagnetic field generated by those currents are also shown.

This thought experiment is inconsistent with the local time independent feature of the jerk amplitudes shown in Fig. 8. Therefore the FACs, i.e., the latitudinally flowing currents are hard to explain the distributions of the jerks seen in Fig. 8 .

5.1.2 Possibility of the ring current as the origin of jerk Figure 10(b) shows a symmetric magnetospheric equatorial ring current. Longitudinally flowing symmetric currents like the ring current can generate geomagnetic field free from local time dependence, and they may be able to explain the local time independent feature shown in Figs. 7, 8 , and 9. Assuming that the westward ring current flows on the dipole equator at four earth radii from the center of the earth, the magnetic field $\delta^{3} \boldsymbol{B}_{r c}=\left(\delta^{3} X_{r c}, \delta^{3} Y_{r c}, \delta^{3} Z_{r c}\right)$ due to the ring current is calculated by the Biot-Savart's law:

$$
\delta^{3} \boldsymbol{B}_{r c}=\frac{\mu_{0} \delta^{3} J}{4 \pi} \oint_{C} \frac{d \boldsymbol{s} \times \hat{\boldsymbol{r}}}{r^{2}},
$$

where $C$ denotes the closed path of the ring current, $J$ is the intensity of the ring current, $\mu_{0}$ is the permeability in the free space, $d s$ is a vector element on the ring current, $\hat{\boldsymbol{r}}$ is the unit vector in a direction from $d \boldsymbol{s}$ to a point on the ground, and $r$ is the distance between them. The contours of $\delta^{3} X_{r c}, \delta^{3} Y_{r c}$, and $\delta^{3} Z_{r c}$ in geographic coordinate system are overlapped with color code in Figs. 7, 8, and 9, respectively. The difference of the color (i.e., red and blue) indicates the difference of the signs of these values, and these signs depend on the direction of the ring current. We discuss the consistency and inconsistency between the distributions of the jerks and those of magnetic field due to the ring current especially from a viewpoint of the jerk amplitudes. The signs of $\delta^{3} X_{r c}$ are the same everywhere on the ground and they are maximum on the dipole equator and minimum at the poles as seen in Fig. 7. The signs of $\delta^{3} X_{n}$ in 1962, $1966,1988,1991$, and 1994 are also the same everywhere on the ground and, at least in the signs, they are consistent with the distributions of $\delta^{3} X_{r c}$. On the other hand, $\delta^{3} X_{n}$ in 1969,1978 , and 1983 have both positive and negative signs and the distributions of those values are, even in the signs, inconsistent with those of $\delta^{3} X_{r c}$.

The effects of the ring current to the $Y$ component (i.e., $\delta^{3} Y_{r c}$ ) appear because the dipole axis tilts from the rotational axis. The distributions of $\delta^{3} Y_{r c}$ have sectorial structures as shown in Fig. 8. $\delta^{3} Y_{r c}$ is zero along the meridian where the plane including both dipole and rotational axes crosses with the surface of the earth. The distributions of $\delta^{3} Y_{n}$ in 1969 and 1978 are very similar to those of $\delta^{3} Y_{r c}$. This correspondence may imply that the jerks have some relations with the currents flowing around the dipole axis. On the other hand, the distributions of $\delta^{3} Y_{n}$ in 1983, 1988, and 1994 do not correspond with those of $\delta^{3} Y_{r c}$.

$\delta^{3} Z_{r c}$ distribution shows a zonal structure as shown in Fig. 9 and it is zero on the geomagnetic equator. The distributions of $\delta^{3} Z_{n}$ are generally consistent with those of $\delta^{3} Z_{r c}$ in 1983, 1988, and 1994, and not in other years.

Here we attempt to clarify whether the distributions of the jerk amplitudes shown in Figs. 7, 8, and 9 can be explained by the abrupt changes in the ring current intensity or not. First an appropriate value of an abrupt change of the ring current intensity, $\delta^{3} \mathrm{~J}$, is determined and then abrupt changes of the geomagnetic field on the ground, $\delta^{3} E_{r c}\left(O_{i}\right)$, due to the abrupt change of the ring current are evaluated at each observatory by the Biot-Savart's law, i.e., Eq. (20).

The value of $\delta^{3} J$ is updated by the Newton method to make the summation of squared residuals

$$
S\left(\delta^{3} J\right)=\sum_{i}\left(\delta^{3} E_{n, h}\left(O_{i}\right)-\delta^{3} E_{r c}\left(O_{i}\right)\right)^{2}
$$

smaller. This procedure is iterated until the optimum value of $\delta^{3} \hat{J}$ is obtained, which makes $S\left(\delta^{3} J\right)$ minimum. Table 1 shows the optimum values of abrupt changes in the ring current (i.e., $\delta^{3} \hat{J}$ ) estimated from the distributions of the jerk amplitudes at midnight for each component. The positive values correspond to eastward changes and negative ones correspond to westward changes. It should be noted that, in 1969, 1978, and 1991, the magnitudes of $\delta^{3} J$ estimated from $\delta^{3} Y$ distributions exceed 10 ampere/year ${ }^{2}$ although those estimated from the distributions of $\delta^{3} X$ or $\delta^{3} Z$ are not so large. The magnitudes of $\delta^{3} J$ in other years are small or almost the same for each component, and the jerks in these years could be of the ring current origin. Therefore it can be concluded that it is difficult for the ring current to generate the observed distribution of jerks in 1969, 1978, and 1991 at least. 

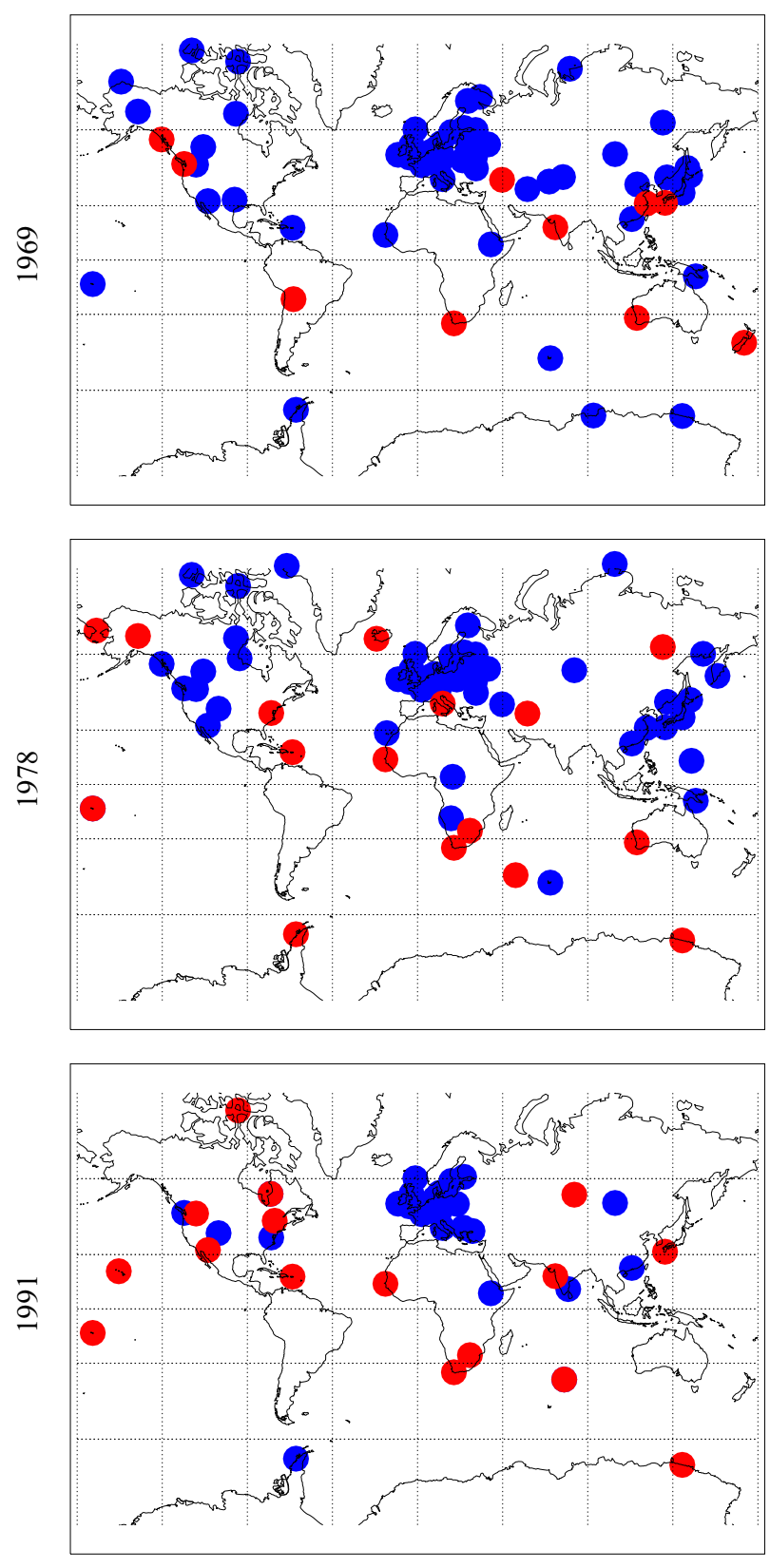

Fig. 11. The global distribution of the occurrence epochs of detected jerks at each observatory for the 1969 (the top panel), the 1978 (the middle panel), and 1991 (the bottom panel) events. The monthly means at 0000-0100LT are used. The blue circles denote early arrivals of jerks before 1970, 1979, and 1992, respectively, and the red ones denote late arrivals of jerks after 1971, 1980, and 1993, respectively.

From the discussion mentioned above, it is difficult for global external currents like the FACs or the ring current to explain the observed jerks in 1969, 1978, and 1991, and it is more natural to consider that these jerks are of internal origin. More detailed analyses of the jerks with more extensive data set are required to investigate the physical mechanisms which can generate the observed jerks.

\subsection{Time lags in the occurrence epochs of 1991 jerk}

It has been reported that the occurrence epochs of jerks are not simultaneous to the global extent but have time lags with a few years. Alexandrescu et al. (1996) showed that the jerks occurred in the southern hemisphere a few years after the occurrences of the 1969 and 1978 jerks in the northern hemisphere. De Michelis et al. (1998) showed that the jerk occurred in North and South America a few years before the occurrence of the 1991 jerk in other regions. The result of our analysis on this topic obtained from 0000-0100 LT data shown in Fig. 11 clearly demonstrates that the jerks occurred in the southern hemisphere except for Antarctica observatories a few years after the occurrences of the 1969 and 1978 jerks in the northern hemisphere. This result is in good agreement with Alexandrescu et al. (1996). We found that the jerk occurred in the southern hemisphere a few years after the occurrence of the 1991 jerk in Europe, which is similar to the time delay for the 1969 and 1978 jerks. These time lags in the occurrence epochs of the 1969, 1978, and 1991 jerks could be due to the mantle conductivity filtering effect (e.g., Backus, 1983; Alexandrescu et al., 1996).

\section{Conclusions}

We analyzed the time series of geomagnetic monthly means of the $X, Y$, and $Z$ components at each local time to clarify the origins and distributions of geomagnetic jerks. Each geomagnetic time series is decomposed into the trend, the seasonal, the stationary AR, and the observational noise components by applying a statistical time series model. The trend component is expressed by a second order spline function because a jerk is an impulse in the third order time derivative of the geomagnetic time series. The model parameters including the positions of the knots of the spline function are estimated by the maximum likelihood method and the number of the knots and the AR order are selected based on the AIC. Distributions of jerk amplitudes are obtained by regarding the optimized positions of the knots as the occurrence epochs of the jerks.

We obtained the following results: The distributions of the geomagnetic jerks are essentially independent of local time. Longitudinally flowing external currents like the ring current may be able to generate such distributions of the jerks while latitudinally flowing external currents like the FACs cannot generate such distributions. If we assume that the ring current is the source of the geomagnetic jerks, the magnitude of the abrupt changes in the ring current intensities estimated from the jerk amplitudes of the $Y$ component exceed, by an order of magnitude, those obtained from the jerk amplitudes of the $X$ or the $Z$ components in 1969, 1978, and 1991. The distributions of the jerks in 1969, 1978, and 1991 are difficult to be explained by the external currents such as the FACs or the ring currents. Therefore it is more plausible that the geomagnetic jerks in 1969, 1978, and 1991 are not of external origin but internal one if we ignore the effects of induced currents due to inhomogeneous conductivity of the crust or the mantle, although the physical mechanism which generates the observed jerks is still unknown.

We also obtain a new result as to the time lags between the occurrence epoch of the 1991 jerk in the northern hemisphere and that in the southern hemisphere. While it is confirmed that the occurrences of the jerks in the southern hemisphere a few years after those of the 1969 and 1978 jerks in the northern hemisphere as pointed out by Alexandrescu $e t$ al. (1996) and De Michelis et al. (1998), it is also found that 
the occurrence of the jerk in the southern hemisphere is a few years after that of the 1991 jerk in Europe. Taking these time lags in occurrence epochs of the jerks into account, the 1969,1978 , and 1991 jerks are confirmed to be global phenomena as suggested by previous papers.

Acknowledgments. We are grateful to staff of Data Analysis Center for Geomagnetism and Space Magnetism, Graduate School of Science, Kyoto University for supplying us the high quality geomagnetic data. We also thank to Y. Yokoyama, S. Ohtani, A. Saito and $\mathrm{G}$. Ueno for the personal discussions and their valuable advice. Miora Mandea and another anonymous referee are also appreciated for reviewing our paper and giving us valuable comments. This research was supported by the Ministry of Education, Science, Sports and Culture (MESSC) of Japan, Grant-in-Aid for Scientific Research on Priority Area "Discovery Science," 10143106, 19982001. Work at the Institute of Statistical Mathematics was in part carried out under the ISM Cooperative Research Program (H12ISM.CRP-2026). The computation in this work has been done using SGI2800 of the Institute of Statistical Mathematics.

\section{References}

Achache, J., V. Courtillot, J. Ducruix, and J.-L. Le Mouël, The late 1960's secular variation impulse: further constraints on deep mantle conductivity, Phys. Earth Planet. Int., 23, 72-75, 1980.

Alexandrescu, M., D. Gibert, G. Hulot, J.-L. Le Mouël, and G. Saracco, Detection of geomagnetic jerks using wavelet analysis, J. Geophys. Res., 100, 12557-12572, 1995.

Alexandrescu, M., D. Gibert, G. Hulot, J.-L. Le Mouël, and G. Saracco, Worldwide wavelet analysis of geomagnetic jerks, J. Geophys. Res., 101, 21975-21994, 1996.

Alldredge, L. R., A discussion of impulses and jerks in the geomagnetic field, J. Geophys. Res., 89, 4403-4412, 1984.

Alldredge, L. R., More on the alleged 1970 geomagnetic jerk, Phys. Earth Planet. Int., 39, 255-264, 1985.

Anderson, B. D. O. and J. B. Moore, Optimal Filtering, Prentice-Hall, 1979.

Backus, G. E., Application of mantle filter theory to the magnetic jerk of 1969, Geophys. J. R. astr. Soc., 74, 713-746, 1983.

Cafarella, L. and A. Meloni, Evidence for a geomagnetic jerk in 1990 across Europe, Annali di Geofisica, XXXVIII, 451-455, 1995.

Courtillot, V., J. Ducruix, and J.-L. Le Mouël, Sur une accélération récente de la variation séculaire du champ magnétique terrestre, $C$. R. Acad. Série D, 1095-1098, 1978.

Davis, R. G. and K. A. Whaler, The 1969 geomagnetic impulse and spin-up of the Earth's liquid core, Phys. Earth Planet. Int., 103, 181-194, 1997.

De Michelis, P., L. Cafarella, and A. Meloni, Worldwide character of the 1991 geomagnetic jerk, Geophys. Res. Lett., 25, 377-380, 1998.

Ducruix, J., V. Courtillot, and J.-L. Le Mouël, The late 1960s secular variation impulse, the eleven year magnetic variation and the electrical conductivity of the deep mantle, Geophys. J. R. astr. Soc., 61, 73-94, 1980 .

Gavoret, J., D. Gibert, M. Menvielle, and J.-L. Le Mouël, Long-term variations of the external and internal components of the earth's magnetic field, J. Geophys. Res., 91, 4787-4796, 1986.
Golovkov, V. P., T. I. Zvereva, and A. O. Simonyan, Common features and differences between "jerks" of 1947, 1958 and 1969, Geophys. Astrophys. Fluid Dyn., 49, 81-96, 1989.

Gubbins, D. and L. Tomlinson, Secular variation from monthly means from Apia and Amberley magnetic observatories, Geophys. J. R. astr. Soc., 86, 603-616, 1986.

Higuchi, T. and S. Ohtani, Automatic identification of large-scale fieldaligned current structures, J. Geophys. Res., 105, 25305-25315, 2000.

Kerridge, D. J. and D. R. Barraclough, Evidence for geomagnetic jerks from 1931 to 1971, Phys. Earth Planet. Int., 39, 228-236, 1985.

Kitagawa, G. and W. Gersch, Smoothness priors analysis of time series, Lecture Notes in Statistics, 116, Springer-Verlag, New York, 1996.

Le Huy, M., M. Alexandrescu, G. Hulot, and J.-L. Le Mouël, On the characteristics of successive geomagnetic jerks, Earth Planets Space, 50, 723-732, 1998.

Le Huy, M., M. Mandea, J.-L. Le Mouël, and A. Pais, Time evolution of the field flow at the top of the core. Geomagnetic jerks, Earth Planets Space, 52, 163-173, 2000.

Le Mouël, J.-L. and V. Courtillot, Core motions, electromagnetic coremantle coupling and variations in the earth's rotation: new constraints from geomagnetic secular variation impulses, Phys. Earth Planet. Int. 24, 236-241, 1981.

Le Mouël, J.-L., J. Ducruix, and C. H. Duyen, The worldwide character of the 1969-1970 impulse of the secular acceleration rate, Phys. Earth Planet. Int., 28, 337-350, 1982.

Macmillan, S., A geomagnetic jerk for the early 1990's, Earth Planet. Sci. Lett., 137, 189-192, 1996.

Malin, S. R. C. and B. M. Hodder, Was the 1970 geomagnetic jerk of internal or external origin?, Nature, 296, 726-728, 1982.

Mandea Alexandrescu, M., D. Gibert, J.-L. Le Mouël, G. Hulot, and G. Saracco, An estimate of average lower mantle conductivity by wavelet analysis of geomagnetic jerks, J. Geophys. Res., 104, 17735-17745, 1999

Mandea, M., E. Bellanger, and J.-L. Le Mouël, A geomagnetic jerk for the end of the 20th century?, Earth Planet. Sci. Lett., 183, 369-373, 2000.

McLeod, M. G., On the geomagnetic jerk of 1969, J. Geophys. Res., 90, 4597-4610, 1985.

McLeod, M. G., Signals and noise in magnetic observatory annual means: mantle conductivity and jerks, J. Geophys. Res., 97, 17261-17290, 1992.

Nevanlinna, H. and C. Sucksdorff, Impulse in global geomagnetic "secular variation”, 1977-1979, J. Geophys., 50, 68-69, 1981.

Press, W. H., B. P. Flannery, S. A. Teukolsky, and W. T. Vetterling, Numerical Recipes: The Art of Scientific Computing, Cambridge University Press, 1986

Stewart, D. N. and K. A. Whaler, Optimal piecewise regression analysis and its application to geomagnetic time series, Geophys. J. Int., 121, 710-724, 1995.

Thompson, D. and J. C. Cain, The geomagnetic jerk of 1969 and the DGRFs, Phys. Earth Planet. Int., 48, 386-388, 1987.

Whaler, K. A., A new method for analysing geomagnetic impulses, Phys. Earth Planet. Int., 48, 221-240, 1987.

H. Nagao (e-mail: nagao@kugi.kyoto-u.ac.jp), T. Iyemori (e-mail: iyemori@kugi.kyoto-u.ac.jp), T. Higuchi (e-mail: higuchi@ism.ac.jp), S. Nakano (e-mail: shiny@kugi.kyoto-u.ac.jp), and T. Araki (e-mail: araki@ kugi.kyoto-u.ac.jp) 